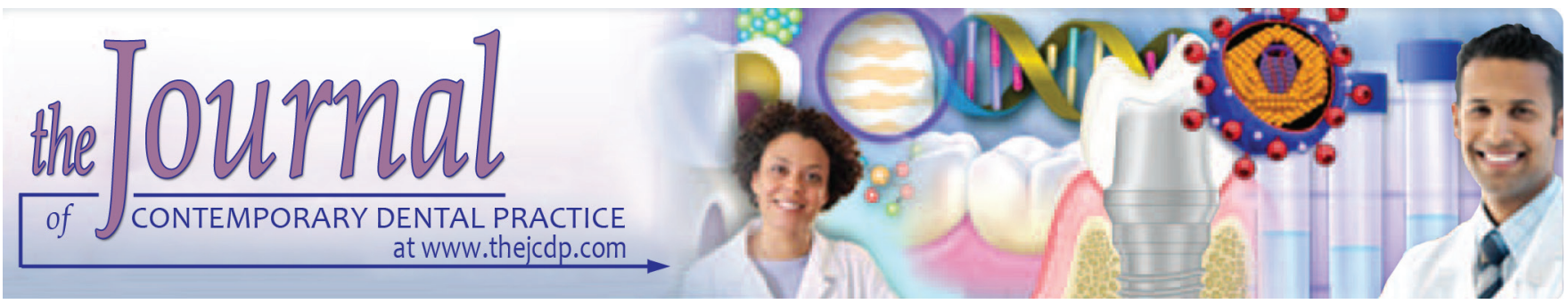

\title{
Root Canal Morphologies of Mesiobuccal Roots of Maxillary Molars using Cone beam Computed Tomography and Periapical Radiographic Techniques in an Iranian Population
}

\footnotetext{
${ }^{1}$ Vahid Zand, ${ }^{2}$ Hadi Mokhtari, ${ }^{3}$ Hamid RM Zonouzi, ${ }^{4}$ Sahand N Shojaei
}

\begin{abstract}
Aim: The aim of this study was to evaluate the accuracy of the results of cone beam computed tomography (CBCT) examinations, confirm the presence or absence of the second mesiobuccal (MB2) root canal in maxillary first and second molars, estimate the distance between the root canal orifices, observe the root types based on Vertucci classification system, and make comparisons between $\mathrm{CBCT}$ and periapical techniques in relation to the identification of the root canal form and the second root canal of the MB root.
\end{abstract}

Materials and methods: The CBCT and periapical radiographic data from the Department of Maxillofacial Radiology were used to select 156 individuals (78 females and 78 males) for the evaluation of the anatomy of maxillary first and second molars.

Results: The prevalence rate of the second root canal in the MB root in the maxillary first molars was $55.1 \%$, with 44.1 and $63.3 \%$ in females and males respectively. The prevalence rate in maxillary second molars was $23.7 \%$, with 15.7 and $30.2 \%$ in females and males respectively. In addition, the longest and shortest distances between the root canal orifices were 2.4 and $0.3 \mathrm{~mm}$ respectively, with a mean of $1.3 \mathrm{~mm}$.

Conclusion: There was a significant difference between the results of periapical and $\mathrm{CBCT}$ radiographic techniques in relation to the presence or absence of the second root canal in the MB roots of maxillary first and second molars $(p<0.01)$, with better results being provided by the $\mathrm{CBCT}$ technique.

Clinical significance: The results of CBCT images about the existence of the second root canal in the MB roots of maxillary first and second molars are more reliable, and we can use this technique in the finding of additional canals in the clinic.

${ }^{1-4}$ Department of Endodontics, Faculty of Dentistry, Tabriz University of Medical Sciences, Tabriz, Iran

Corresponding Author: Hadi Mokhtari, Department of Endodontics, Faculty of Dentistry, Tabriz University of Medical Sciences, Tabriz, Iran, Phone: +989143131992, e-mail: mokhtari.z.h@gmail.com
Keyword: Cone beam computed tomography, Dental radiography, Root canal therapy.

How to cite this article: Zand V, Mokhtari H, Zonouzi HRM, Shojaei SN. Root Canal Morphologies of Mesiobuccal Roots of Maxillary Molars using Cone beam Computed Tomography and Periapical Radiographic Techniques in an Iranian Population. J Contemp Dent Pract 2017;18(9):745-749.

Source of support: Nil

Conflict of interest: None

\section{INTRODUCTION}

Proper root canal treatment involves cleaning, shaping, and obturation of the root canal with a suitable material. Proper and sufficient knowledge about the morphology of the root canal system has a great role in the success of the treatment. Of all the reasons for the failure of root canal treatment, a lack of proper knowledge about the anatomy of the pulp can be mentioned, and mistakes in the diagnosis and treatment planning are less important. ${ }^{1}$

Since accessory and extra root canals are common phenomena, and their incomplete treatment is one of the reasons for the failure of root canal treatment, acquiring proper knowledge about the morphology and anatomy of the root canal system and its variations is of utmost importance. $^{2}$

Morphological variations have been reported in relation to the anatomy of the root canal system in different ethnic populations; ${ }^{3-6}$ therefore, it is necessary to gain knowledge about the anatomy of this system in different ethnic groups for successful endodontic treatment.

Different techniques have been used for the investigation of the anatomy of the root canal system, including conventional and digital radiographic systems, ${ }^{7}$ staining and cleaning, ${ }^{8-10}$ and computed tomography (CT) 
techniques. ${ }^{11}$ The introduction of the CBCT system led to a revolution in the craniofacial imaging. The advantages of this imaging system include its nondestructive nature, three-dimensional (3D) reconstruction of the root canal system, the ability to restrict the imaging area, which, in turn, decreases patient radiation dose, an increase in the accuracy and resolution of images, and a decrease in image artifacts. ${ }^{12-16}$

The main characteristic of CBCT is that several views in multiple planes are provided by one rotational scanning to present volumetric data and images that are related to each other. Therefore, this technique has the capacity to yield a 3D reconstruction of the anatomic structures of the root and root canal system, and it is possible to analyze the images with a software program without interference with the principal format of digital imaging and communications in medicine. ${ }^{17}$

The capacity of CBCT to eliminate superimposition of the surrounding structures and yield a 3D image of the root has made it the best-imaging technique for the evaluation of the morphology of the root canal system. In addition, compared with the staining and clearing techniques, it does not damage the tooth structure and yields the results of evaluations in a shorter time.

Based on a literature review, no study is available on the comparison of $\mathrm{CBCT}$ and periapical radiographic techniques in relation to the anatomy of the mesiobuccal (MB) roots of maxillary first and second molars in Iranian populations, especially in Tabriz. Therefore, the present study was undertaken to evaluate the accuracy of the CBCT imaging technique in relation to the presence or absence of a second root canal in the mesial roots of maxillary first and second molars, measure the distance between the root canal orifices, identify the root canal types based on the Vertucci classification, ${ }^{11}$ and compare the results with those of the periapical radiographic technique.

\section{MATERIALS AND METHODS}

A total of 156 patients (78 males and 78 females) were selected for the evaluation of the anatomy of maxillary first and second molars based on the CBCT and periapical radiographic data available in the Department of Maxillofacial Radiology, Tabriz Faculty of Dentistry.
The images were provided using the NewTom GI CBCT (Verona/Italy) unit in the Department of Oral and Maxillofacial Department, Tabriz Faculty of Dentistry. The unit delivers a cone-shaped X-ray beam and has a flat-panel detector with a resolution of 1,536 × 1,920 pixels, a pixel of $127 \times 127\left(\mu^{2}\right)$, a pixel depth of 14 bits, a rotation of $360^{\circ}$, a scan time of 18 seconds, and a $\mathrm{kVp}$ of 110. The initial and final reconstructions were carried out with NNT viewer (version 2:17) software program. The exposure conditions were selected automatically.

The CBCT data were processed by NNT viewer software program to yield images that were displayed on a 19-inch liquid crystal display monitor (PHILIPs, 190B) with a resolution of $1,024 \times 1,208$ pixels and 32 bits in a dimly lit room by one observer. The images were used to provide $0.5-\mathrm{mm}$-thick axial cross sections. A radiologist and an endodontist evaluated the root canal morphologies based on Vertucci classification (Fig. 1) on CBCT and periapical radiographs. When no agreement was reached on a case, it was excluded from the study.

In this study, patient gender and the side on which the maxillary first and second molars were located and also the root canal types of these teeth and the distances between the orifices of MB1 of MB2 root canals were determined. The available periapical radiographs were used to evaluate whether it was possible to identify the presence of a second root canal in the MB roots and the distance between its orifice and the orifice of the MB1 root canal.

\section{Statistics Analysis}

Data were analyzed with Statistical Package for the Social Sciences version 19. Fisher's exact test was used to compare CBCT and periapical radiographs; t-test was used to compare qualitative and quantitative variables. Chi-squared test was used to compare quantitative and qualitative values. Statistical significance was set at $p<0.05$.

\section{RESULTS}

The prevalence of the second root canal in the MB root (MB2) of maxillary first molars was 55.11\%. The root canal types of the $\mathrm{MB}$ roots of maxillary first molars were as follows: Type I, $43.6 \%$; type II, $10.3 \%$; type III, $44.2 \%$; type IV, $0.6 \%$; and type V, $1.3 \%$. These types in females

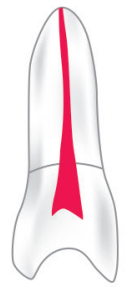

Type I(1)

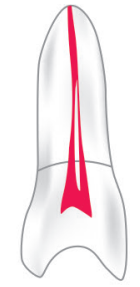

Type II(2-1)

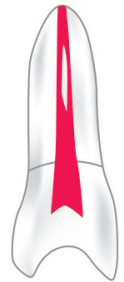

Type III(1-2-1)

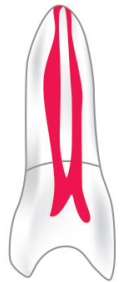

Type IV(2)
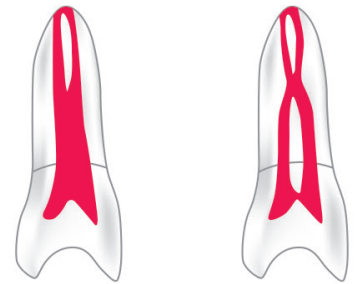

Type V(1-2)

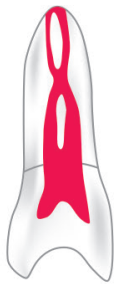

Type VII(1-2-1-2)

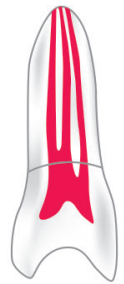

Type VIII(3)

Fig. 1: Vertucci classification 
were as follows: Type I, 55.7\%; type II, 8.6\%; Type III, $32.9 \%$; and Type $\mathrm{V}, 2.9 \%$; the rates in males were as follows: Type I, 33.7\%; Type II, 11.6\%; Type III, 53.5\%; Type IV, $1.2 \%$. In female individuals, $41.4 \%$ of the root canals exhibited MB2 root canal and male individuals exhibited a rate of $63.3 \%$. In female individuals, $51.4 \%$ of maxillary first molars on the left side and $48.6 \%$ on the right side exhibited MB2 root canal; in male individuals, $48.8 \%$ of maxillary first molars on the left side and $51.2 \%$ of these teeth on the right side exhibited MB2 root canal. In relation to the maxillary side, the first molars on the left side exhibited the following root canal types: Type I, $39.7 \%$; type II, $12.8 \%$; type III, $47.4 \%$; the prevalence rates were as follows in the right side: Type I, $47.4 \%$; type II, $7.7 \%$; type III, $41 \%$; type IV, $1.3 \%$; and type V, 2.6\%. In relation to the relationship between the prevalence of MB2 root canals in the first molars and the maxillary side, the prevalence rates on the left and right sides were $57.7 \%$ and $52.6 \%$ respectively. The mean distance between the two root canal orifices, with 82 teeth of 156 teeth having separate orifices, was $1.3 \mathrm{~mm}$, with a range of 0.3 to $2.4 \mathrm{~mm}$.

In maxillary second molars, the prevalence of MB2 root canal was $23.7 \%$, with the following prevalence rates for root canal types: Type I, $77.6 \%$; type II, 3.2\%; type III, $17.3 \%$; type IV, $0.6 \%$; and type V, $1.3 \%$. The root canal types in female individuals were as follows: Type I, $87.1 \%$ and type III, $12.9 \%$; these rates in males were as follows: Type I, $69.8 \%$; type II, $5.8 \%$; type III, $20.9 \%$; type IV, $1.2 \%$; and type $\mathrm{V}, 2.3 \%$. The prevalence rates of MB2 root canals in female and male individuals were $15.7 \%$ and $30.2 \%$ respectively. The prevalence rates of root canal types on the left side were: Type I, 75.6\%; type II, 5.1\%; type III, $17.9 \%$; type V, $1.3 \%$. On the right side, these rates were as follows: Type I, 79.5\%; type II, $1.3 \%$; type III, $16.7 \%$; type IV, $1.3 \%$; and type $\mathrm{V}, 1.3 \%$. In relation to the side, the prevalence rates of MB2 root canals on the right and left sides were $21.8 \%$ and $25.6 \%$ respectively. The orifice of the MB2 root canal was present in 36 teeth, with a mean distance of $1.3 \mathrm{~mm}$ between the two orifices, and a range of 0.3 to $2.4 \mathrm{~mm}$ (Tables 1 and 2).

Table 1: Comparison of the results between $\mathrm{CBCT}$ and periapical radiographic techniques for maxillary first molars

\begin{tabular}{llll}
\hline & $\begin{array}{l}\text { The number } \\
\text { of MB2 root } \\
\text { number of } \\
\text { samples }\end{array}$ & $\begin{array}{l}\text { canals in } \\
\text { maxillary first } \\
\text { molars }\end{array}$ & Percentages \\
\hline $\begin{array}{l}\text { Periapical } \\
\begin{array}{l}\text { Cone beam computed } \\
\text { tomography }\end{array}\end{array}$ & 156 & 5 & 0.0320 \\
\hline $\begin{array}{l}\text { The results in relation to maxillary second molars were significantly } \\
\text { different between the CBCT and periapical radiographic techniques } \\
(p<0.01)\end{array}$ & 86 & 0.5512 \\
\hline
\end{tabular}

\section{DISCUSSION}

Since the presence of extra root canals is a common occurrence, and their incomplete treatment is one of the reasons for treatment failure, it is necessary to acquire proper and sufficient knowledge about the number of roots and root canals and their anatomic variations. It should be pointed out that the root canal morphology might be different depending on ethnicity in different countries.

In the present study on an Iranian population in Tabriz, the prevalence of MB2 root canals in maxillary first molars was $55.1 \%$ and based on Vertucci classification, type I (43.6\%) and type II (44.2\%) had the highest prevalence rates, which is different from those of a study in India, in which the prevalence rates of root canal morphologies of MB2 root canals in maxillary first molars was as follows: Type I, 39.7\%; type II, $12.8 \%$; type III, $47.4 \%$; the prevalence rates were as follows on the right side: Type I, $69 \%$; type II, $24 \%$; and type IV, $0.4 \%{ }^{18}$

In a study on first molars in a Korean population, $34.4 \%$ of the root canals were type I, with $40.65 \%$ and $20.45 \%$ being type IV and type II respectively, as the highest prevalence rates and type II and type V with 0.25 and $2 \%$ as the lowest prevalence rates. ${ }^{19}$

In a study on maxillary first molars in a Chinese population, of 612 molar teeth evaluated, 423 teeth were type I, with $45,22,61$, and 60 teeth being type II, type III, type $\mathrm{IV}$, and type $\mathrm{V}$ respectively. ${ }^{20}$

In another study on first molars in an Iranian population, $46.1 \%$ of the root canals were type I and more than $50 \%$ of the MB roots of maxillary first molars had more than one root canal; therefore, based on the ethnicity, the root canal morphologies of maxillary first molars are different in different populations.

In the present study, in maxillary second molars, the prevalence of MB2 root canal was $23.7 \%$, with $15.7 \%$ in females and $30.2 \%$ in males. The prevalence rates of root canal types were as follows: Type I, 39.7\%; type II, $12.8 \%$; type III, $47.4 \%$; the prevalence rates were as follows on the right side: Type I, $77.6 \%$; type II, $3.2 \%$; type III, $17.30 \%$; type IV, $0.61 \%$; and type $\mathrm{V}, 1.3 \%$. Similar to the maxillary

Table 2: Comparison of the results between CBCT and periapical radiographic techniques for maxillary second molars

\begin{tabular}{llll}
\hline & \multicolumn{3}{c}{$\begin{array}{l}\text { The number } \\
\text { of MB2 root } \\
\text { canals in } \\
\text { maxillary first }\end{array}$} \\
& $\begin{array}{l}\text { Total } \\
\text { number of } \\
\text { samples }\end{array}$ & $\begin{array}{l}\text { molars } \\
\text { morcentages }\end{array}$ \\
\hline $\begin{array}{l}\text { Periapical } \\
\begin{array}{l}\text { Cone beam computed } \\
\text { tomography }\end{array}\end{array}$ & 156 & 2 & 0.0128 \\
\hline
\end{tabular}

The results in relation to maxillary second molars were significantly different between the $\mathrm{CBCT}$ and periapical radiographic techniques $(p<0.01)$ 
first molars, the root canal morphology of second molars is different in different ethnicities.

In an Indian population, the prevalence rates of root canals in maxillary second molars were as follows: Type I, $39.7 \%$; type II, $12.8 \%$; type III, $47.4 \%$; the prevalence rates were as follows on the right side: Type I, $80.6 \%$; type II, $15.3 \%$; type IV, $2.7 \%$; and type V, $1.4 \%{ }^{12}$ In a Korean population, the prevalence rates were as follows: Type I, $57.4 \%$; type II, $2.1 \%$; type III, $2.1 \%$ and type IV, $8.5 \% .{ }^{13}$ In a Chinese population, of 382 maxillary second molars, 329 teeth exhibited type I, 16 teeth were type II, 9 teeth were type III, 10 teeth exhibited type IV, 16 teeth were type V, and 1 tooth was type VIII. ${ }^{20}$

In the present study, the prevalence of MB2 root canals in maxillary second molars was $15.7 \%$ in females and $30.2 \%$ in males; however, in a Chinese population, the prevalence of MB2 root canals was $10.64 \%$ in females, with $16.15 \%$ in males. In the Korean population, these rates were $32 \%$ and $36.72 \%$ in females and males respectively. ${ }^{19,20}$

In the present study, the prevalence rates of MB2 root canals in maxillary second molars on the left and right sides were 15.7 and $30.2 \%$ respectively. In the Chinese population, these prevalence rates on the left and right sides were 13.24 and $13.64 \%$ respectively. In the Korean population, the prevalence rates were 35.84 and $32.93 \%$ respectively. ${ }^{19-21}$

Based on the data presented above, it is not possible to reach a conclusion in relation to the prevalence of MB2 root canals in maxillary second molars because the effect of ethnicity is very strong in this respect. In relation to the distance between the orifices of MB1 and MB2 root canals in both first and second upper molars, the minimum and maximum distances were 0.3 and $2.4 \mathrm{~mm}$ respectively, with a mean distance of $1.3 \mathrm{~mm}$.

Comparison of the results of $\mathrm{CBCT}$ and periapical radiographic techniques in relation to the presence or absence of MB2 root canals in maxillary first and second molars showed the superiority of the CBCT technique, and since diagnostic periapical radiographs were used in the present study, it was very difficult to visualize MB2 root canals. It is obvious that use of radiographs taken with tube angulation can be very effective in the evaluation of the presence or absence of MB2 root canals.

\section{CONCLUSION}

Based on the results of the present study, use of CBCT technique is more reliable for the evaluation of the morphology of maxillary first and second molars.

\section{CLINICAL SIGNIFICANCE}

The results of CBCT images about existence of the second root canal in the $\mathrm{MB}$ roots of maxillary first and second molars are more reliable, and we can use this technique in the finding of additional canals in the clinic.

\section{REFERENCES}

1. Walton, RE.; Torabinejad, M. Principle and practice of endodontics. 3rd ed. Philadelphia (PA): W.B Sounders; 2000. p. 16-18, 167, 216.

2. Vertucci FJ. Root canal morphology and its relationship to endodontic procedures. Endod Topics 2005 Mar;10(1):3-29.

3. Sert S, Bayirli GS. Evaluation of the root canal configurations of the mandibular and maxillary permanent teeth by gender in the Turkish population. J Endod 2004 Jul;30(6):391-398.

4. Rwenyonyi CM, Kutesa A, Muwazi LM, Buwembo W. Root and canal morphology of mandibular first and second permanent molar teeth in an Ugandan population. Odontology 2009 Aug;97(2):92-96.

5. Chen G, Yao H, Tong C. Investigation of the root canal configuration of mandibular first molars in a Taiwan Chinese population. Int Endod J 2009 Nov;42(11):1044-1049.

6. Weng XL, Yu SB, Zhao SL, Wang HG, Mu T, Tang RY, Zhou XD. Root canal morphology of permanent maxillary teeth in the Han nationality in Chinese Guanzhong area: A new modified root canal staining technique. J Endod 2009 May;35(5): 651-656.

7. Patel S, Dawood A, Whaites E, Pitt Ford T. New dimensions in endodontic imaging: part 1. Conventional and alternative radiographic systems. Int Endod J 2009 Jun;42(6):447-462.

8. Awawdeh L, Abdullah H, Al-Qudah A. Root form and canal morphology of Jordanian maxillary first premolars. J Endod 2008 Aug;34(8):956-961.

9. Naoum HJ, Love RM, Chandler NP, Herbison P. Effect of $\mathrm{X}$-ray beam angulation and intraradicular contrast medium on radiographic interpretation of lower first molar root canal anatomy. Int Endod J 2003 Jan;36(1):12-19.

10. Thomas RP, Moule AJ, Bryant R. Root canal morphology of maxillary permanent first molar teeth at various ages. Int Endod J 1993 Sep;26(5):257-267.

11. Mokhtari H, Niknami M, Mokhtari Zonouzi HR, Sohrabi A, Ghasemi N, Golzar AA. Accuracy of cone beam computed tomography in determining the root canal morphology of mandibular first molars. Iran Endod J 2016 Spring;11(2): 101-105.

12. Fan B, Yang J, Gutmann JL, Fan M. Root canal systems in mandibular first premolars with C-shaped root configurations. Part I: microcomputed tomography mapping of the radicular groove and associated root canal cross-sections. J Endod 2008 Nov;34(11):1337-1341.

13. SalarPour M, Farhad Mollashahi N, Mousavi E, SalarPour E. Evaluation of the effect of tooth type and canal configuration on crown size in mandibular premolars by cone beam computed tomography. Iran Endod J 2013 Autumn;8(4):153-156.

14. Sberna MT, Rizzo G, Zacchi E, Cappare P, Rubinacci A. A preliminary study of the use of peripheral quantitative computed tomography for investigating root canal anatomy. Int Endod J 2009 Jan;42(1):66-75.

15. Cotton TP, Geisler TM, Holden DT, Schwartz SA, Schindler WG. Endodontic application of cone beam volumetric tomography. J Endod 2007 Sep;33(9):1121-1132.

16. Scarfe WC, Farman AG, Sukovic P. Clinical applications of cone beam computed tomography in dental practice. J Can Dent Assoc 2006 Mar;72(1):75-80.

17. Scarfe WC, Levin MD, Gane D, Farman AG. Use of cone beam computed tomography in endodontics. Int J Dent 2009;2009:634567. 
18. Singh S, Pawar M. Root canal morphology of South Asian Indian maxillary molar teeth. Euro J Dent 2015 Jan-Mar;9(1): 133-144.

19. Kim Y, Lee SJ, Woo J. Morphology of maxillary first and second molar analyzed by cone beam computed tomography in a Korean population: variations in the number of roots and canals and the incidence of fusion. J Endod 2012 Aug;38(8):1063-1068.
20. Jing YN, Ye X, Liu DG, Zhang ZY, Ma XC. Cone beam computed tomography was used for study of root and canal morphology of maxillary first and second molars. Beijing Da Xue Bao 2014 Dec;46(6):958-962.

21. Rouhani R, Bagherpour A, Akbari M, Azizi M, Nejat A, Naghavi N. Cone beam computed tomography evaluation of maxillary first and second molars in Iranian population: a morphological study. Iran Endod J 2014 Summer;9(3):190-194. 